\title{
HUREWICZ IMAGES IN BP AND THE ARF-KERVAIRE INVARIANT
}

\author{
VICTOR P. SNAITH \\ Faculty of Mathematical Studies, University of Southampton, Highfield, \\ Southampton SO17 1BJ, England \\ e-mail:vps@maths.soton.ac.uk
}

(Received 14 October, 1999; revised 26 April, 2001)

\begin{abstract}
In this paper $B P$-theory is used to give a proof that there exists a stable homotopy element in $\pi_{2^{n+1}-2}^{S}\left(\mathbf{R} P^{\infty}\right)$ with non-zero Hurewicz image in $j u$-theory if and only if there exists an element of $\pi_{2^{n+1}-2}^{S}\left(S^{0}\right)$ that is represented by a framed manifold of Arf invariant one.
\end{abstract}

2000 Mathematics Subject Classification. 55P42, 55N20.

\section{Introduction.}

1.1. Deciding whether or not framed manifolds with non-trivial Arf-Kervaire invariant exist is one of the longstanding problems in homotopy theory. In terms of stable homotopy theory this is concerned with the existence of certain two-primary classes in the stable homotopy group $\pi_{m}^{S}\left(S^{0}\right)$. It was shown in [4] that the problem only remains when $m=2^{n+1}-2$. In fact calculations similar to those we are about to do give the result of [4] very easily.

At the moment it is known only that such framed manifolds exist when $n=1,2,3,4,5$.

It is convenient to study an equivalent problem. When $m>0$ there is a split surjection, called the Kahn-Priddy map, of the form

$$
\pi_{m}^{S}\left(\mathbf{R} P^{\infty}\right) \longrightarrow \pi_{m}^{S}\left(S^{0}\right) \otimes \mathbf{Z}_{2}
$$

and when $m=2^{n+1}-2$ the condition that $\theta \in \pi_{2^{n+1}-2}^{S}\left(\mathbf{R} P^{\infty}\right)$ maps to a stable homotopy element represented by a framed manifold of Arf invariant one modulo 2 is equivalent to the Steenrod operation, $S q^{2^{n}}: H^{2^{n}-1}(C(\theta) ; \mathbf{Z} / 2) \longrightarrow H^{2^{n+1}-1}(C(\theta) ; \mathbf{Z} / 2)$, being non-trivial on the mod 2 cohomology of the mapping cone, $C(\theta)$, of $\theta$.

The main result (Theorem 4.2) of this paper is to show that this happens if and only if the $j u$-theory Hurewicz homomorphism, $H_{j u}: \pi_{2^{n+1}-2}^{S}\left(\mathbf{R} P^{\infty}\right) \longrightarrow j u_{2^{n+1}-2}\left(\mathbf{R} P^{\infty}\right)$ is non-trivial on $[\theta]$. This result was first conjectured a long while ago by Barratt and Mahowald, appearing in print in [3]. The proof given by Klippenstein and me in [8] unfortunately contains a gap. Namely the group in [8, Lemma 3.1] is slightly larger than claimed, allowing some ruinous indeterminacy into the argument. In 1990 Knapp pointed out the problem and Klippenstein tried to repair the mistake, failing to do so before he left the academic profession. A few years later Knapp produced a correct proof [9] based on work of Miller-Ravenel-Wilson [10]. In view of its history one cannot expect a non-technical proof of this conjecture but I believe that the proof given here is 'elementary' in the sense that it proceeds via a series of generalised 
homology calculations which are rather standard. Firstly Theorem 4.2 is reduced to a result in $J_{*}^{\prime}$ (Theorem 3.5), which is a generalised homology theory constructed from $B P$-theory. Then the proof of Theorem 3.5 requires only a basic familiarity with the rather awkward formula for the canonical anti-automorphism in $B P$-theory, discovered by Quillen and described in [1] and [16]. After that the proof rests on some easily obtained formulae involving binomial coefficients modulo 2 and a two-step induction argument.

In the past I was inclined to give $B P_{*}$ a wide berth. Therefore I am particularly grateful to Huajian Yang for introducing me to Quillen's formulae in a very userfriendly manner. Huajian was my Britton post-doctoral research assistant at McMaster University before he, too, left the academic profession.

Here is an outline of the contents of the paper. In $\$ 2$ and $\$ 3$ I recapitulate the facts and formulae that are needed about $B P$ and $\mathbf{R} P^{\infty}$ and then introduce the homology theories $J_{*}$ and $J_{*}^{\prime}$, which are to $B P$ and $B P \wedge B P$ what $j u$ is to $b u$. The crux of the paper is to restrict the possibilities for Hurewicz images in these theories by analysing the canonical anti-involution induced by switching the factors in $B P \wedge B P$. This is done in Theorem 3.5. Theorem 3.4, which is a weaker and easier result, is stated without proof. The latter may be proved by a similar induction to Theorem 3.5 using $b u \wedge B P$ rather than $B P \wedge B P$. In $\S 4$ the deduction of Theorem 4.2 from Theorem 3.5 is explained. In $\S 5$ an induction argument is given as a first step towards proving Theorem 3.5. In order to get around the point at which the argument of $\S 5$ falters we derive in $\$ 6$ some complicated combinatorial identities modulo 2 which must be satisfied by the coefficients in our hypothetical Hurewicz image. The coefficients in question are either 0 or 1 . In $\$ 7$ it is shown how, by studying the combinatorial identities of $\S 6$ in low degrees, one can complete the induction argument for the proof of Theorem 3.5.

\section{2. $B P$-theories and $\mathbf{R} P^{\infty}$.}

2.1. Let $B P$ denote the 2-adic Brown-Peterson spectrum ([1, pp. 109-116]; [16]) whose homotopy, $\pi_{*}(B P)=B P_{*}$, is isomorphic to $\mathbf{Z}_{2}\left[v_{1}, v_{2}, v_{3}, \ldots\right]$ where $\mathbf{Z}_{2}$ denotes the 2-adic integers and $\operatorname{deg}\left(v_{i}\right)=2\left(2^{i}-1\right)$. Then we have $B P^{*}\left(\mathbf{C} P^{\infty}\right) \cong$ $B P^{*}[[x]]$, where $B P^{*}=B P_{-*}$ and $\operatorname{deg}(x)=2$. The series [2] $x \in B P^{*}[[x]]$ is defined by [2] $x=f^{*}(x)$, where $f: \mathbf{C} P^{\infty}=B S^{1} \longrightarrow \mathbf{C} P^{\infty}$ is induced by the squaring map on the circle, $S^{1}$. From [16, Lemma 3.17, p. 20] we have

$$
[2] x \equiv 2 x+\sum_{i \geq 1} v_{i} x^{2^{i}}\left(\text { modulo }<2, v_{1}, v_{2}, v_{3}, \ldots>^{2} B P^{*}[[x]]\right) .
$$

Now consider $B P^{*}\left(\mathbf{R} P^{2 t}\right) \cong B P^{*} \oplus \tilde{B P^{*}}\left(\mathbf{R} P^{2 t}\right)$. The composition of $f$ with the canonical map, $i: \mathbf{R} P^{2 t} \longrightarrow \mathbf{C} P^{2 t} \longrightarrow \mathbf{C} P^{\infty}$, is trivial. Also $x^{t+1}$ is zero in $\tilde{B P^{*}}\left(\mathbf{C} P^{2 t}\right)$ and there is an induced isomorphism of the form

$$
i^{*}: B P^{*}[[x]] /<x^{t+1},[2] x>\stackrel{\cong}{\longrightarrow} B P^{*}\left(\mathbf{R} P^{2 t}\right) .
$$

This isomorphism is proved together with the assertion that every element of $\tilde{B P^{2 m}}\left(\mathbf{R} P^{2 t}\right)$ may be written uniquely as (the image under $i^{*}$ of) $\sum_{I, j} \epsilon_{I} v^{I} x^{j}$, where the sum is taken over all sequences of non-negative integers, $I=\left(i_{1}, \ldots, i_{r}\right)$, $v^{I}=v_{1}^{i_{1}} \ldots v_{r}^{i_{r}}, 2 j-\sum_{s=1}^{r} i_{s} 2\left(2^{s}-1\right)=2 m$ and $1 \leq j \leq t$ with each $\epsilon_{I}=0$ or 1 . To 
prove both assertions one observes that the Atiyah-Hirzebruch spectral sequence for the reduced group, $\tilde{B P}^{*}\left(\mathbf{R} P^{2 t}\right)$, collapses because it is concentrated in even total degree. The $E_{2}$-term is generated by $B P^{*}$ and $x$ so that $i^{*}$ is surjective. Also $E_{2}^{p, q}$ is zero unless $0<p \leq 2 t$ is even and $q=2 n$, in which case it is isomorphic to $B P^{2 n} \otimes \mathbf{Z} / 2$. Therefore the order of $\tilde{B P}^{2 m}\left(\mathbf{R} P^{2 t}\right)$ is $2^{a}$, where $a=a_{1}+\ldots+a_{t}$ and $a_{j}$ is equal to the number of sequences $I$ such that $2 j-\sum_{s=1}^{r} i_{s} 2\left(2^{s}-1\right)=2 m$. This is also the number of expressions of the form $\sum_{I, j} \epsilon_{I} v^{I} x^{j}$ in dimension $2 \mathrm{~m}$. On the other hand, the form of [2] $x$ shows that every element of $\tilde{B P}^{2 m}\left(\mathbf{R} P^{2 t}\right)$ may be written in at least one way in the desired form. Hence, by counting group orders, this expression must be unique and $i^{*}$ must be an isomorphism.

The S-dual of $\mathbf{R} P^{2 t}$ is homotopy equivalent to $\Sigma^{1-2^{i}} \mathbf{R} P^{2^{i}-2} / \mathbf{R} P^{2^{i}-2 t-2}$ for $i$ sufficiently large, by [5, pp. 205-208], and the previous discussion yields short exact sequences of the form

$$
\begin{aligned}
0 \longrightarrow & B P^{2^{i}-2 h}\left(\mathbf{R} P^{2^{i}-2} / \mathbf{R} P^{2^{i}-2 t-2}\right) \\
& \longrightarrow B P^{2^{i}-2 h}\left(\mathbf{R} P^{2^{i}-2}\right) \longrightarrow B P^{2^{i}-2 h}\left(\mathbf{R} P^{2^{i}-2 t-2}\right) \longrightarrow 0 .
\end{aligned}
$$

In addition, we have S-duality isomorphisms [5] of the form

$$
\begin{aligned}
B P^{2^{i}-2 h}\left(\mathbf{R} P^{2^{i}-2} / \mathbf{R} P^{2^{i}-2 t-2}\right) & \cong B P^{1-2 h}\left(\Sigma^{1-2^{i}} \mathbf{R} P^{2^{i}-2} / \mathbf{R} P^{2^{i}-2 t-2}\right) \\
& \cong B P_{2 h-1}\left(\mathbf{R} P^{2 t}\right) .
\end{aligned}
$$

For $1 \leq h \leq t$ the element $x^{2^{i-1}-h} \in B P^{2^{i}-2 h}\left(\mathbf{R} P^{2^{i}-2}\right)$ maps to zero in $B P^{2^{i}-2 h}\left(\overline{\mathbf{R}} P^{2^{i}-2 t-2}\right)$ and we may define $x_{2 h-1} \in B P_{2 h-1}\left(\mathbf{R} P^{2 t}\right)$ to be equal to the image of $x^{2^{i-1}-h} \in B P^{2^{i}-2 h}\left(\mathbf{R} P^{2^{i}-2} / \mathbf{R} P^{2^{i}-2 t-2}\right)$ under the $\mathrm{S}$-duality isomorphism.

Every element of $\tilde{B P^{2^{i}}-2 s}\left(\mathbf{R} P^{2^{i}-2} / \mathbf{R} P^{2^{i}-2 t-2}\right)$ can be expressed uniquely in the form $\sum_{I, j} \epsilon_{I} v^{I} x^{j}$ with $2^{i-1}-t \leq j \leq 2^{i-1}-1$ and each $\epsilon_{I} \in\{0,1\}$. Hence every element of $\tilde{B P}_{2 s-1}\left(\mathbf{R} P^{2 t}\right)=B P_{2 s-1}\left(\mathbf{R} P^{2 t}\right)$ is uniquely expressible in the form $\sum_{I, k} \epsilon_{I} V^{I} x_{2 k+1}$ with $1 \leq 2 k+1 \leq 2 t-1$ and each $\epsilon_{I} \in\{0,1\}$. The relation that $x^{2^{i-1}-h-1} \cdot[2] x=0$ translates into a congruence of the form

$$
2 x_{2 h-1}+\sum_{j \geq 1} v_{j} x_{2 h-2^{j+1}+1} \equiv 0\left(\text { modulo }<2, v_{1}, v_{2}, v_{3}, \ldots>^{2} B P_{*}\left(\mathbf{R} P^{2 t}\right)\right) .
$$

Recall [1, p. 89] that if $X$ is a commutative ring spectrum with unit, $\iota: S^{0} \longrightarrow X$, there are two maps, $\eta_{L}=1 \wedge \iota$ and $\eta_{R}=\iota \wedge 1$, from $X$ to $X \wedge X$ that give $\pi_{*}(X \wedge X)=(X \wedge X)_{*}$ the structure of a left or right $\pi_{*}(X)$-module, respectively. When $X=B P$ there exist canonical elements, $t_{i} \in(B P \wedge B P)_{2\left(2^{i}-1\right)},[\mathbf{1}$, Theorem 16.1 p. 112], [16, Theorem 3.11 p. 17] such that $(B P \wedge B P)_{*} \cong B P_{*}\left[t_{1}, t_{2}, t_{3}, \ldots\right]$ as a left $B P_{*}$-module. From the collapsed Atiyah-Hirzebruch spectral sequence for $(B P \wedge B P)_{*}\left(\mathbf{R} P^{2 t}\right)$ there is an isomorphism of left $B P_{*}$-modules of the form

$$
(B P \wedge B P)_{*}\left(\mathbf{R} P^{2 t}\right) \cong(B P \wedge B P)_{*} \otimes_{B P_{*}} B P_{*}\left(\mathbf{R} P^{2 t}\right) \cong B P_{*}\left(\mathbf{R} P^{2 t}\right)_{*}\left[t_{1}, t_{2}, t_{3}, \ldots\right]
$$

Therefore every element of $(B P \wedge B P)_{2 s-1}\left(\mathbf{R} P^{2 t}\right)$ is uniquely expressible in the form $\sum_{I, I^{\prime}, k} \epsilon_{I} v^{I} t^{I^{\prime}} x_{2 k+1}$, with $1 \leq 2 k+1 \leq 2 t-1$ and each $\epsilon_{I} \in\{0,1\}$. Here $t^{I^{\prime}}=t^{\left(i_{1}^{\prime}, \ldots . i_{r}^{\prime}\right)}$ denotes $t_{1}^{i_{1}^{\prime}} \ldots t_{r}^{i_{r}^{\prime}}$. 
3. $J_{*}$ and $J_{*}^{\prime}$

3.1. Let $\psi^{3}: B P \longrightarrow B P$ denote the Adams operation in $B P$-theory ([1, Part II]; [11], [13, pp. 59-60]). Hence $\psi^{3}$ is equal to multiplication by $3^{k}$ on $B P_{2 k}$ and by $3^{j+1}$ on $B P_{2 j+1}\left(\mathbf{R} P^{2 t}\right)$. The last fact follows easily from the formula $\psi^{3}(x)=3^{-1} x[13$, Corollary 4.3 p. 60] since the S-duality isomorphism is given by slant product with the $B P$-Thom class of the tangent bundle of $\mathbf{R} P^{2 t}\left[\mathbf{1}\right.$, p. 264] and since $\psi^{3}$ commutes with slant products. It also follows that $\psi^{3} \wedge \psi^{3}: B P \wedge B P \longrightarrow B P \wedge B P$ is given by multiplication by $3^{k}$ on $(B P \wedge B P)_{2 k}$ and by $3^{j+1}$ on $(B P \wedge B P)_{2 j+1}\left(\mathbf{R} P^{2 t}\right)$.

Define spectra $J$ and $J^{\prime}$ by the following cofibration sequences

$$
J \stackrel{\pi}{\longrightarrow} B P \stackrel{\psi^{3}-1}{\longrightarrow} B P \stackrel{\pi_{1}}{\longrightarrow} \Sigma J
$$

and

$$
J^{\prime} \stackrel{\pi^{\prime}}{\longrightarrow} B P \wedge B P \stackrel{\psi^{3} \wedge \psi^{3}-1}{\longrightarrow} B P \wedge B P \stackrel{\pi_{1}^{\prime}}{\longrightarrow} \Sigma J^{\prime}
$$

Since $\psi^{3} \cdot \iota=\iota: S^{0} \longrightarrow B P, \eta_{L}$ and $\eta_{R}$ induce maps $\tilde{\eta}_{L}, \tilde{\eta}_{R}: J \longrightarrow J^{\prime}$, respectively. Also $\iota$ induces a (unique) map, $\tilde{\iota}: S^{0} \longrightarrow J$, such that $\pi \cdot \tilde{\iota}=\iota$.

Let $n \geq 1$ be an integer. Since $\psi^{3}-1$ is injective on $B P_{2^{n+1}-2}\left(\mathbf{R} P^{2^{n+1}}\right) \cong B P_{2^{n+1}-2}$, there is an isomorphism of the form

$$
\left(\pi_{1}\right)_{*}: B P_{2^{n+1}-1}\left(\mathbf{R} P^{2^{n+1}}\right) \otimes \mathbf{Z} / 2^{n+2} \stackrel{\cong}{\longrightarrow} J_{2^{n+1}-2}\left(\mathbf{R} P^{2^{n+1}}\right),
$$

since $3^{2^{n}}-1=2^{n+2}(2 s+1)$. Similarly there is an isomorphism of the form

$$
\left(\pi_{1}^{\prime}\right)_{*}:(B P \wedge B P)_{2^{n+1}-1}\left(\mathbf{R} P^{2^{n+1}}\right) \otimes \mathbf{Z} / 2^{n+2} \stackrel{\cong}{\longrightarrow} J_{2^{n+1}-2}^{\prime}\left(\mathbf{R} P^{2^{n+1}}\right) .
$$

By means of the isomorphisms, $\left(\pi_{1}\right)_{*}$ and $\left(\pi_{1}^{\prime}\right)_{*}$, we may represent elements of $J_{2^{n+1}-2}\left(\mathbf{R} P^{2^{n+1}}\right)$ and $J_{2^{n+1}-2}^{\prime}\left(\mathbf{R} P^{2^{n+1}}\right)$ by sums in degree $2^{n+1}-1$ of the form $\sum_{I, k} \epsilon_{I} v^{I} x_{2 k+1}$ and $\sum_{I, I^{\prime}, k} \epsilon_{I} v^{I} t^{I^{\prime}} x_{2 k+1}$, respectively, as in $\S 2.1$.

Now let $T: B P \wedge B P \longrightarrow B P \wedge B P$ be the map which interchanges the factors. Then $T_{*}=c$, the conjugation, on $(B P \wedge B P)_{*}(X)$. In $\S 5.2$ we shall need the following formulae for $c\left(v_{k}\right)=\left(\eta_{R}\right)_{*}\left(v_{k}\right)$. Recall that $B P_{*}$ embeds, via the Hurewicz homomorphism, into $H_{*}\left(B P ; \mathbf{Z}_{2}\right) \cong \mathbf{Z}_{2}\left[m_{1}, m_{2}, \ldots\right]$, where $\operatorname{deg}\left(m_{i}\right)=\operatorname{deg}\left(v_{i}\right)$ and $v_{i}=$ $2 m_{i}-\sum_{j=1}^{i-1} m_{j} v_{i-j}^{2^{j}}$.

Lemma 3.2. Let $I=<2, v_{1}, v_{2}, \ldots>\triangleleft B P_{*}=\mathbf{Z}_{2}\left[v_{1} . v_{2}, \ldots\right]$. Then, for $k \geq 1$,

$$
\left(\eta_{R}\right)_{*}\left(v_{k}\right)=2 t_{k}+\sum_{j \geq 1}^{k} v_{j} t_{k-j}^{2^{j}}\left(\text { modulo } I^{2}\left[t_{1}, t_{2}, \ldots\right]\right)
$$

in $(B P \wedge B P)_{*} \cong \mathbf{Z}_{2}\left[v_{1}, v_{2}, \ldots, t_{1}, t_{2}, t_{3}, \ldots\right]$.

Proof. We use the formulae of [1, Theorem 16.1, p. 112] and [16, Theorem 3.11 p. 17] from which we see that $\left(\eta_{R}\right)_{*}\left(v_{1}\right)=2 t_{1}+v_{1}$ and that in $(B P \wedge B P)_{*} \otimes \mathbf{Q}_{2}$ $\left(\eta_{R}\right)_{*}\left(m_{k}\right)=\sum_{j=0}^{k} m_{j} t_{k-j}^{j}$. The results follows by induction on $k$. 
3.3. Let $E$ be a commutative ring spectrum and let $(\eta)_{*}: B P^{*}\left(\mathbf{C} P^{\infty}\right) \longrightarrow$ $(E \wedge B P)^{*}\left(\mathbf{C} P^{\infty}\right)$ denote the map induced by $\eta$, the unit of $E$. When $E=B P$, $(B P \wedge B P)^{*}\left(\mathbf{C} P^{\infty}\right) \cong(B P \wedge B P)_{-*}[[x]]$, where $x=\left(\eta_{L}\right)_{*}(x)$ in dimension two. On the other hand, $\eta=\eta_{R}$, so that the formula of [1, Lemma 6.3, p. 60] and [16, Lemma 1.51 , p. 9] becomes

$$
c(x)=\sum_{v \geq 0} b_{v}^{B P} x^{v+1} \in(B P \wedge B P)^{2}\left(\mathbf{C} P^{\infty}\right) .
$$

This formula also holds in $(B P \wedge B P)^{2}\left(\mathbf{R} P^{\infty}\right)$.

Since $\left(\eta_{L}\right)_{*}\left(x_{2 k+1}\right) \in(B P \wedge B P)_{*}\left(\mathbf{R} P^{2^{n+1}}\right)$ corresponds under S-duality to $x^{2^{i-1}-k-1}$ in

$$
(B P \wedge B P)^{*}\left(\mathbf{R} P^{2^{i}-2} / \mathbf{R} P^{2^{i}-2^{n+1}-2}\right) \cong\left(\frac{x^{2^{i-1}-2^{n}} \mathbf{Z}_{2}\left[v_{1}, v_{2}, \ldots, t_{1}, t_{2}, \ldots\right][[x]]}{x^{2^{i-1}} \mathbf{Z}_{2}\left[v_{1}, v_{2}, \ldots, t_{1}, t_{2}, \ldots\right][[x]]}\right) /([2] x)
$$

(where this isomorphism follows from the canonical form for elements that was discussed in $\$ 2.1$ ), it follows that

$$
c\left(\left(\eta_{L}\right)_{*}\left(x_{2 k+1}\right)\right)=\left(\eta_{R}\right)_{*}\left(x_{2 k+1}\right)=\sum_{w=0}^{k} b_{k, w}\left(\eta_{L}\right)_{*}\left(x_{2 w+1}\right),
$$

where

$$
\sum_{w=0}^{k} b_{k, w} x^{2^{i-1}-w-1}=\left(\sum_{v \geq 0} b_{v}^{B P} x^{v+1}\right)^{2^{i-1}-k-1}
$$

in $(B P \wedge B P)^{*}\left(\mathbf{R} P^{2^{i}-2} / \mathbf{R} P^{2^{i}-2^{n+1}-2}\right)$. The coefficients $b_{v}^{B P} \in(B P \wedge B P)_{*} \cong \mathbf{Z}_{2}\left[v_{1}\right.$, $\left.v_{2}, \ldots, t_{1}, t_{2}, \ldots\right]$ satisfy [16, Theorem 3.11 (proof), p. 17 and Theorem 1.48(c) p. 8]

$$
\sum_{i \geq 0}\left(\eta_{L}\right)_{*}\left(m_{i}\right)=\sum_{s \geq 0}\left(\eta_{R}\right)_{*}\left(m_{s}\right)\left(\sum_{v \geq 0} b_{v}^{B P}\right)^{2^{s}} .
$$

This equation holds in $\mathbf{Q}_{2}\left[v_{1}, v_{2}, \ldots, t_{1}, t_{2}, \ldots\right]$ but setting each $v_{i}$ to zero we obtain the equation

$$
0=\sum_{i \geq 0} t_{i}\left(\sum_{v \geq 0} b_{v}^{B P}\right)^{2^{i}}
$$

and this equation holds in $\mathbf{Z}_{2}\left[v_{1}, v_{2}, \ldots, t_{1}, t_{2}, \ldots\right] /<v_{1}, v_{2}, \ldots>$. Hence we find that

$$
0=\sum_{i \geq 0} t_{i} \sum_{v \geq 0}\left(b_{v}^{B P}\right)^{2^{i}} \in \mathbf{Z}_{2}\left[v_{1}, v_{2}, \ldots, t_{1}, t_{2}, \ldots\right] /<2, v_{1}, v_{2}, \ldots>
$$

Since $t_{0}=1=b_{0}$ one sees by induction that $b_{v}^{B P} \in<2, v_{1}, v_{2}, \ldots>$

$\mathbf{Z}_{2}\left[v_{1}, v_{2}, \ldots, t_{1}, t_{2}, \ldots\right]$ except when $v=2^{m}-1$ for some $m$ and for each $v \geq 1$ 


$$
0 \equiv \sum_{j=0}^{v} t_{j}\left(b_{2^{v-j}-1}^{B P}\right)^{2^{j}}\left(\text { modulo }<2, v_{1}, v_{2}, \ldots>\mathbf{Z}_{2}\left[v_{1}, v_{2}, \ldots, t_{1}, t_{2}, \ldots\right]\right)
$$

which we shall use in proving Lemma 6.2.

Now we can state our main technical results.

THEOREM 3.4. In the notation of $\$ \S 2.1$ and 3.1, let $u \in J_{2^{n+1}-2}\left(\boldsymbol{R} P^{2^{n+1}}\right)$ be represented as $u=\sum_{I} \epsilon_{I} v^{I} x_{2^{n+1}-\operatorname{deg}\left(v^{I}\right)-1}$. If

$$
\left(\tilde{\eta}_{R}\right)_{*}(u)=\left(\tilde{\eta}_{L}\right)_{*}(u) \in J_{2^{n+1}-2}^{\prime}\left(\mathbf{R} P^{2^{n+1}}\right),
$$

then either for some $0 \leq d \leq n+1$ and $\epsilon=1$ or for some $d \geq n+2$ and $\epsilon=0$ we have

$$
u=\epsilon 2^{d} x_{2^{n+1}-1}+\sum_{l\left(I^{\prime}\right) \geq d+1} \epsilon_{I^{\prime}} v^{I^{\prime}} x_{2^{n+1}-\operatorname{deg}\left(v^{I^{\prime}}\right)-1} .
$$

Here the length of $I=\left(i_{1}, \ldots, i_{t}\right)$ is defined to be equal to $l(I)=i_{1}+\ldots+i_{t}$.

In $§ 7.1$ we shall improve Theorem 3.4 to the following more difficult result.

THEOREM 3.5. In the notation of $\S \S 2.1$ and 3.1 , let $u \in J_{2^{n+1}-2}\left(\boldsymbol{R} P^{2^{n+1}}\right)$ be represented as

$$
u=\epsilon 2^{n+1} x_{2^{n+1}-1}+\sum_{l\left(I^{\prime}\right) \geq n+2} \epsilon_{I^{\prime}} v^{I^{\prime}} x_{2^{n+1}-\operatorname{deg}\left(v^{I^{\prime}}\right)-1}
$$

and satisfy

$$
\left(\tilde{\eta}_{R}\right)_{*}(u)=\left(\tilde{\eta}_{L}\right)_{*}(u) \in J_{2^{n+1}-2}^{\prime}\left(\mathbf{R} P^{2^{n+1}}\right),
$$

where $\epsilon, \epsilon_{I^{\prime}} \in\{0,1\}$. Then $\epsilon_{I^{\prime}}=0$ if $l\left(I^{\prime}\right)=n+2$.

REMARK 3.6. Theorem 3.4 may be proved directly by mapping to $b u \wedge B P$, which amounts to setting $v_{j}=0$ for all $j \geq 2$ and then following a (simpler) version of the induction which proves Proposition 5.3. Alternatively one may derive Theorem 3.4 from Theorem 3.5 by replacing $u$ by $2^{n+1-d} u$.

\section{4. $\operatorname{Im}(J)$-theory and the Kervaire invariant.}

4.1. Suppose that $\theta: S^{2^{n+1}-2} \longrightarrow \mathbf{R} P^{\infty}$ is an S-map whose mapping cone is denoted by $C(\theta)$. In dimension $2^{n+1}-2$ the Kahn-Priddy map [6] gives a split surjection of stable homotopy groups

$$
\pi_{2^{n+1}-2}^{S}\left(\mathbf{R} P^{\infty}\right) \longrightarrow \pi_{2^{n+1}-2}^{S}\left(S^{0}\right) \otimes \mathbf{Z}_{2}
$$

onto the 2-Sylow subgroup of the stable homotopy groups of spheres. The Kervaire invariant ([3], [4], [7]) of a framed manifold yields a homomorphism from $\pi_{2^{n+1}-2}^{S}\left(S^{0}\right) \otimes \mathbf{Z}_{2}$ to the group of order two. Furthermore, it is well-known that the 
image of $[\theta] \in \pi_{2^{n+1}-2}^{S}\left(\mathbf{R} P^{\infty}\right)$ has non-trivial Kervaire invariant if and only if the Steenrod operation ([12], [15])

$$
S q^{2^{n}}: H^{2^{n}-1}(C(\theta) ; \mathbf{Z} / 2) \cong \mathbf{Z} / 2 \longrightarrow H^{2^{n+1}-1}(C(\theta) ; \mathbf{Z} / 2)
$$

is non-trivial.

Now let $b u$ denote 2-adic connective K-theory and define $j u$-theory by means of the fibration $j u \longrightarrow b u \stackrel{\psi^{3}-1}{\longrightarrow} b u$. Hence $j u_{*}$ is a generalised homology theory for which $j u_{2^{n+1}-2}\left(\mathbf{R} P^{\infty}\right) \cong \mathbf{Z} / 2^{n+2}$. Recall that, if $\iota \in j u_{2^{n+1}-2}\left(S^{2^{n+1}-2}\right) \cong \mathbf{Z}_{2}$ is a choice of generator, the associated $j u$-theory Hurewicz homomorphism

$$
H_{j u}: \pi_{2^{n+1}-2}^{S}\left(\mathbf{R} P^{\infty}\right) \longrightarrow j u_{2^{n+1}-2}\left(\mathbf{R} P^{\infty}\right) \cong \mathbf{Z} / 2^{n+2}
$$

is defined by $H_{j u}([\theta])=\theta_{*}(\iota)$.

We are now ready to state the main result of this paper.

THEOREM 4.2. For $n \geq 1$ the image of $[\theta] \in \pi_{2^{n+1}-2}^{S}\left(\boldsymbol{R} P^{\infty}\right)$ under the ju-theory Hurewicz homomorphism

$$
H_{j u}([\theta]) \in j u_{2^{n+1}-2}\left(\mathbf{R} P^{\infty}\right) \cong \mathbf{Z} / 2^{n+2}
$$

is non-trivial if and only if $S q^{2^{n}}$ is non-trivial on $H^{2^{n}-1}(C(\theta) ; Z / 2)$.

In any case, $2 H_{j u}([\theta])=0$.

Proof. Consider the following commutative diagram.

$$
\begin{aligned}
& J_{2^{n+1}-2}\left(S^{2^{n+1}-2}\right) \longrightarrow J_{2^{n+1}-2}\left(\mathbf{R} P^{\infty}\right) \\
& B P_{2^{n+1}-1}(C(\theta)) \longrightarrow B P_{2^{n+1}-2}\left(S^{2^{n+1}-2}\right) \\
& \left|\psi^{3}-1 \quad\right| \psi^{3}-1 \\
& B P_{2^{n+1}-1}\left(\mathbf{R} P^{\infty}\right) \longrightarrow B P_{2^{n+1}-1}(C(\theta)) \longrightarrow B P_{2^{n+1}-2}\left(S^{2^{n+1}-2}\right) \\
& \left.\right|_{J_{2^{n+1}-2}\left(\mathbf{R} P^{\infty}\right) \longrightarrow J_{2^{n+1}-2}(C(\theta))}
\end{aligned}
$$


Let $\tilde{\iota} \in J_{2^{n+1}-2}\left(S^{2^{n+1}-2}\right)$ be the class given by the J-theory unit as in $\S 3.1$. The J-theory Hurewicz image is given by $\theta_{*}(\tilde{\iota}) \in J_{2^{n+1}-2}\left(\mathbf{R} P^{\infty}\right) \cong J_{2^{n+1}-2}\left(\mathbf{R} P^{2^{n+1}}\right)$. It is an element satisfying the conditions of Theorems 3.4 and 3.5. The image of $\tilde{\imath}$ in $B P_{2^{n+1}-2}\left(S^{2^{n+1}-2}\right)$ is $\iota$ of $\S 3.1$, which lifts to $\iota^{\prime \prime} \in B P_{2^{n+1}-1}(C(\theta))$. Then $\left(\psi^{3}-1\right)\left(\iota^{\prime \prime}\right)$ lifts to $\alpha \in B P_{2^{n+1}-1}\left(\mathbf{R} P^{\infty}\right)$ and, by [14, Proposition 2, pp. 241-242], the image of $\alpha$ in $J_{2^{n+1}-2}\left(\mathbf{R} P^{2^{n+1}}\right)$ is equal to $\theta_{*}(\tilde{\iota})$. Therefore, by Theorem 3.4 , we obtain an equation of the form

$$
\psi^{3}\left(\iota^{\prime \prime}\right)=\iota^{\prime \prime}+\epsilon 2^{d} x_{2^{n+1}-1}+\sum_{l\left(I^{\prime}\right) \geq d+1} \epsilon_{I^{\prime}} v^{I^{\prime}} x_{2^{n+1}-\operatorname{deg}\left(v^{I^{\prime}}\right)-1}+2^{n+2} \beta \in B P_{2^{n+1}-1}(C(\theta))
$$

for some $0 \leq d \leq n+1, \epsilon=0,1$ and $\beta \in B P_{2^{n+1}-1}\left(\mathbf{R} P^{\infty}\right)$.

Define $j u$ by the 2-local cofibring, $j u \longrightarrow b u \stackrel{\psi^{3}-1}{\longrightarrow} b u$, so that $j u_{2^{n+1}-2}\left(\mathbf{R} P^{\infty}\right) \cong$ $\mathbf{Z} / 2^{n+2}$, generated by $\lambda_{*}\left(x_{2^{n+1}-1}\right)$, where $\lambda_{*}: J_{*}(X) \longrightarrow j u_{*}(X)$ is induced by the canonical map $\lambda: B P \rightarrow b u$. Therefore the $j u$-theory Hurewicz image of $\theta$ is $\epsilon 2^{d} \lambda_{*}\left(x_{2^{n+1}-1}\right)$.

First we must show that $d \geq n+1$, which will imply that the $j u$-theory Hurewicz image of $\theta$ is trivial unless $d=n+1$ and in that case is non-trivial if and only if $\epsilon=1$. If $\epsilon=1$ and $d<n+1$, we replace $\iota$ by $2^{n+1-d} \iota$. Then the argument which is to follow shows that $2^{n+1-d} \theta$ is detected by $S q^{2^{n}}$ on the mod 2 cohomology of its mapping cone. This is easily seen to be impossible, by comparing the mapping cone sequences for $2^{n+1-d} \theta$ and $2^{n-d} \theta$.

The fact that $d \geq n+1$ implies that $2 H_{j u}([\theta])=0$.

Now write $s_{n}: B P_{*}(X) \longrightarrow B P_{*-2 n}(X)$ for the Landweber-Novikov operation in $B P$-homology corresponding to $s_{(n, 0,0, \ldots)}$ in $[\mathbf{1}, \mathrm{p}$. 12]. We are going to study the consequences of the relation, $3^{m} \psi^{3} s_{m}=s_{m} \psi^{3}$. This relation is established by observing that the sum of the left and right sides over $m$ correspond to two ring operations in $B P$-cohomology and therefore are equal if and only if these cohomology operations agree on $x \in B P^{2}\left(\mathbf{C} P^{\infty}\right)$, which is easily verified. In addition, if $T: B P \longrightarrow H \mathbf{Z} / 2$ corresponds to the Thom class, then a similar argument shows that $\left(S q^{2 m}\right)_{*} T_{*}=T_{*} s_{m}: B P_{*}(X) \longrightarrow H_{*-2 m}(X ; \mathbf{Z} / 2)$.

Also, if $0 \leq m \leq t$, using the formulae of [1, Part I, $\S 5$ and $\S 8.1]$ it is not difficult to show (we shall only need this formula modulo 2 ) that

$$
s_{m}\left(x_{2 t+1}\right)=(-1)^{m}\left(\begin{array}{c}
m+t \\
m
\end{array}\right) x_{2 t-2 m+1} \in B P_{2 t-2 m+1}\left(\mathbf{R} P^{\infty}\right) .
$$

Bearing in mind the previous discussion about what to do if $d<n+1$, we may suppose that $d=n+1$ and write

$$
\psi^{3}\left(\iota^{\prime \prime}\right)=\iota^{\prime \prime}+\epsilon 2^{n+1} x_{2^{n+1}-1}+2^{n+2} \beta+\gamma \in B P_{2^{n+1}-1}(C(\theta)) .
$$

Here $\beta \in B P_{2^{n+1}-1}\left(\mathbf{R} P^{\infty}\right) \subset B P_{2^{n+1}-1}(C(\theta))$ and, by Theorem 3.5,

$$
\gamma=\sum_{l\left(I^{\prime}\right)>n+2} \epsilon_{I^{\prime}} v^{I^{\prime}} x_{2^{n+1}-\operatorname{deg}\left(v^{I^{\prime}}\right)-1}
$$

Applying the relation with $m=2^{n-1}$ we obtain the following equation in $B P_{2^{n}-1}(C(\theta)) \cong B P_{2^{n}-1}\left(\mathbf{R} P^{\infty}\right)$ : 


$$
s_{2^{n-1}}\left(\iota^{\prime \prime}\right)+\epsilon 2^{n+1}\left(\begin{array}{c}
2^{n-1}+2^{n}-1 \\
2^{n-1}
\end{array}\right) x_{2^{n}-1}+2^{n+2} s_{2^{n-1}}(\beta)+s_{2^{n-1}}(\gamma)=3^{2^{n-1}} 3^{2^{n-1}} s_{2^{n-1}}\left(\iota^{\prime \prime}\right)
$$

because $\psi^{3}$ acts like multiplication by $3^{2^{n-1}}$ on $B P_{2^{n}-1}\left(\mathbf{R} P^{\infty}\right)$.

We are going to apply

$$
\lambda_{*}: B P_{2^{n+1}-1}\left(\mathbf{R} P^{\infty}\right) \longrightarrow b u_{2^{n+1}-1}\left(\mathbf{R} P^{\infty}\right) \quad\left(\operatorname{modulo} 2^{n+3}\right)
$$

to the above equation, bearing in mind that $\lambda_{*}\left(v_{k}\right)=0$ for $k \geq 2$ and that

$$
0=v_{1} x_{2 j-1}+2 x_{2 j+1} \in b u_{2 j+1}\left(\mathbf{R} P^{\infty}\right) .
$$

In $b u_{*}\left(\mathbf{R} P^{\infty}\right)$ consider $\lambda_{*}\left(s_{m}\left(v_{k}\right)\right) x_{2 j+1}$. If $m \neq 2^{k}-1,2^{k}-2$ then $\lambda_{*}\left(s_{m}\left(v_{k}\right)\right)$ is a multiple of $v_{1}^{2+e}$, for some $e \geq 0$, and therefore $\lambda_{*}\left(s_{m}\left(v_{k}\right)\right) x_{2 j+1} \in 4 b u_{2 j+2^{k+1}-1-2 m}\left(\mathbf{R} P^{\infty}\right)$. Similarly one sees that $\lambda_{*}\left(s_{2^{k}-2}\left(v_{k}\right)\right) x_{2 j+1} \in 2 b u_{2 j+3}\left(\mathbf{R} P^{\infty}\right)$. Also, since $s_{2^{k}-1}$ cannot decrease Adams filtration, $\lambda_{*}\left(s_{2^{k}-1}\left(v_{k}\right)\right) \in 2 b u_{0}\left(S^{0}\right)$ and $\lambda_{*}\left(s_{2^{k}-1}\left(v_{k}\right)\right) x_{2 j+1} \in$ $2 b u_{2 j+1}\left(\mathbf{R} P^{\infty}\right)$.

Now consider $v_{i_{1}} v_{i_{2}} \ldots v_{i_{t}} x_{2 j+1} \in B P_{2^{n+1}-1}\left(\mathbf{R} P^{\infty}\right)$ and

$$
\lambda_{*}\left(s_{2^{n-1}}\left(v_{i_{1}} v_{i_{2}} \ldots v_{i_{t}} x_{2 j+1}\right)\right)=\sum_{a_{1}+\ldots a_{t+1}=2^{n-1}} \lambda_{*}\left(s_{a_{1}}\left(v_{i_{1}}\right)\right) \ldots \lambda_{*}\left(s_{a_{t}}\left(v_{i_{t}}\right)\right) \lambda_{*}\left(s_{a_{t+1}}\left(x_{2 j+1}\right)\right) .
$$

The discussion above shows that this lies in $2^{t} b u_{2^{n}-1}\left(\mathbf{R} P^{\infty}\right)$ unless $t=0$. Also $\lambda_{*}\left(s_{2^{n-1}}\left(x_{2^{n+1}-1}\right)\right) \in 2 b u_{2^{n}-1}\left(\mathbf{R} P^{\infty}\right)$, since

$$
\left(\begin{array}{c}
2^{n-1}+2^{n}-1 \\
2^{n-1}
\end{array}\right)=2(2 s+1)
$$

for some $s$. Hence both $2^{n+2} \lambda_{*}\left(s_{2^{n-1}}(\beta)\right)$ and $\lambda_{*}\left(s_{2^{n-1}}(\gamma)\right)$ lie in $2^{n+3} b u_{2^{n}-1}\left(\mathbf{R} P^{\infty}\right)$.

From this discussion, in the previous notation, our equation implies the following congruence in $b u_{2^{n}-1}\left(\mathbf{R} P^{\infty}\right) \cong \mathbf{Z} / 2^{2^{n-1}}$ :

$$
\left(3^{2^{n}}-1\right) \lambda_{*}\left(s_{2^{n-1}}\left(\iota^{\prime \prime}\right)\right) \equiv 2^{n+2} \epsilon \text { modulo } 2^{n+3} b u_{2^{n}-1}\left(\mathbf{R} P^{\infty}\right) .
$$

However, for $n \geq 1,\left(3^{2^{n}}-1\right)=2^{n+2}(2 w+1)$, for some $w$, so that $\epsilon=1$ if and only if $\lambda_{*}\left(s_{2^{n-1}}\left(\iota^{\prime \prime}\right)\right)$ is a generator of $b u_{2^{n}-1}\left(\mathbf{R} P^{\infty}\right)$. The factorisation, $T: B P \stackrel{\lambda}{\longrightarrow} b u \longrightarrow$ $H \mathbf{Z} / 2$, implies that $\epsilon=1$ if and only if the dual Steenrod operation, $S q_{*}^{2^{n}}$, is nontrivial on $H_{2^{n+1}-1}(C(\theta) ; \mathbf{Z} / 2)$, which is equivalent to $S q^{2^{n}}$ being non-trivial on $H^{2^{n}-1}(C(\theta) ; \mathbf{Z} / 2)$. This completes the proof.

\section{Theorem 3.5 - the induction step}

5.1. In this section we begin the proof of Theorem 3.5, establishing the main part of an induction argument.

Consider once more the isomorphism

$$
(B P \wedge B P)^{*}\left(\mathbf{R} P^{2^{i}-2} / \mathbf{R} P^{2^{i}-2^{n+1}-2}\right) \cong\left(\frac{x^{2^{i-1}-2^{n}} \mathbf{Z}_{2}\left[v_{1}, v_{2}, \ldots, t_{1}, t_{2}, \ldots\right][[x]]}{x^{2^{i-1}} \mathbf{Z}_{2}\left[v_{1}, v_{2}, \ldots, t_{1}, t_{2}, \ldots\right][[x]]}\right) /([2] x)
$$


established in $\S 3.3$ by means of the canonical form for elements, discussed in $\S 2.1$. Recall that there are isomorphisms of the form

$$
\begin{aligned}
& (B P \wedge B P)^{2^{i}-2^{n+1}}\left(\mathbf{R} P^{2^{i}-2} / \mathbf{R} P^{2^{i}-2^{n+1}-2}\right) \otimes \mathbf{Z} / 2^{n+2} \\
& \cong(B P \wedge B P)_{2^{n+1}-1}\left(\mathbf{R} P^{2^{n+1}}\right) \otimes \mathbf{Z} / 2^{n+2} \\
& \cong J_{2^{n+1}-2}^{\prime}\left(\mathbf{R} P^{2^{n+1}}\right) .
\end{aligned}
$$

Suppose that $u \in J_{2^{n+1}-2}\left(\mathbf{R} P^{2^{n+1}}\right)$ is represented by

$$
u=\epsilon 2^{n+1} x_{2^{n+1}-1}+\sum_{l\left(I^{\prime}\right) \geq n+2} \epsilon_{I^{\prime}} V^{I^{\prime}} x_{2^{n+1}-\operatorname{deg}\left(v^{I^{\prime}}\right)-1}
$$

with $\epsilon, \epsilon_{I^{\prime}} \in\{0,1\}$. If $u$ satisfies $\left(\tilde{\eta}_{R}\right)_{*}(u)=\left(\tilde{\eta}_{L}\right)_{*}(u) \in J_{2^{n+1}-2}^{\prime}\left(\mathbf{R} P^{2^{n+1}}\right)$ we wish to show that $\epsilon_{I^{\prime}}$ is zero for all $I^{\prime}$ with $l\left(I^{\prime}\right)=n+2$. For this purpose we shall compute in $(B P \wedge B P)^{2^{i}-2^{n+1}}\left(\mathbf{R} P^{2^{i}-2} / \mathbf{R} P^{2^{i}-2^{n+1}-2}\right) \otimes \mathbf{Z} / 2^{n+2}$ or rather in a (graded) quotient, denoted by $E^{*}$ for brevity.

Let $I=<2, v_{1}, v_{2}, \ldots>\triangleleft B P_{*}=\mathbf{Z}_{2}\left[v_{1}, v_{2}, \ldots\right]$ be the ideal generated by $2, v_{1}$, $v_{2}, \ldots$ According to $\$ 2.1$, since [2] $x=0$, we have, for all $j \geq 0,2 x^{1+j} \equiv \sum_{i \geq 1} v_{i} x^{2^{i}+j}$ (modulo $\left.I^{2}[[x]]\right)$ in $B P_{*}[[x]] /([2] x)$. By induction on $d \geq 0$ we have, for all $j \geq 0$,

$$
\left.2^{d+1} x^{d+1+j} \equiv \sum_{i_{1}, \ldots, i_{d+1} \geq 1} v_{i_{1}} \ldots v_{i_{d+1}} x^{2^{i_{1}}+2^{i_{2}}+\ldots+2^{i_{d+1}+j}} \text { (modulo } I^{d+2}[[x]]\right) .
$$

Similarly, since $i$ is much larger than $n$, we have, in $(B P \wedge B P)^{*}\left(\mathbf{R} P^{2^{i}-2} / \mathbf{R} P^{2^{i}-2^{n+1}-2}\right)$,

$$
\begin{gathered}
2^{n+2} v^{I} t^{I^{\prime}} x^{2^{i-1}-k-1}=\left(\sum_{i_{1}, \ldots, i_{n+2} \geq 1} v_{i_{1}} \ldots v_{i_{n+2}} x^{\left.2^{i_{1}}+2^{i_{2}}+\ldots+2^{i_{n+2}-n-2}\right) v^{I} t^{I^{\prime}} x^{2^{i-1}-k-1}}\right. \\
+\sum_{l\left(I^{\prime \prime}\right) \geq n+3, I^{\prime \prime \prime}, j} \epsilon_{I^{\prime \prime}}^{I^{\prime \prime}} t^{I^{\prime \prime \prime}} x^{j}
\end{gathered}
$$

Therefore, if we set

$$
u_{n}=\sum_{i_{1}, \ldots, i_{n+2} \geq 1} v_{i_{1}} \ldots v_{i_{n+2}} x^{i^{i_{1}}+2^{i_{2}}+\ldots+2^{i_{n+2}}-n-2},
$$

then

$$
E^{*}=\left(\frac{x^{2^{i-1}-2^{n}} \mathbf{Z}_{2}\left[v_{1}, v_{2}, \ldots, t_{1}, t_{2}, s\right][[x]]}{x^{2^{i-1}} \mathbf{Z}_{2}\left[v_{1}, v_{2}, \ldots, t_{1}, t_{2}, \ldots\right][[x]]}\right) / \approx
$$

where $\approx$ denotes the ideal generated by elements of the forms [2] $x, v^{I} t^{I^{\prime}} x^{2^{i-1}-2^{n}}$, $l(I) \geq n+3$ and $u_{n} t^{I^{\prime \prime}} x^{2^{i-1}-2^{n}-(n+2)}$. Then $E^{*}$ is a quotient of the required form. Let

$$
\rho:\left(\frac{x^{2^{i-1}-2^{n}} \mathbf{Z}_{2}\left[v_{1}, v_{2}, \ldots, t_{1}, t_{2}, s\right][[x]]}{x^{2^{i-1}} \mathbf{Z}_{2}\left[v_{1}, v_{2}, \ldots, t_{1}, t_{2}, \ldots\right][[x]]}\right) /([2] x) \otimes \mathbf{Z} / 2^{n+2} \longrightarrow E^{*}
$$

denote the canonical quotient map. 
Consider the (graded) subgroup $D^{*} \subseteq E^{*}$, generated by the elements of the form $\rho\left(v^{I} t^{I^{\prime}} x^{2^{i-1}-k-1}\right)$ with $l(I)=n+2$. By induction, using the canonical form of $\S 2.1$ for elements of $(B P \wedge B P)^{*}\left(\mathbf{R} P^{2^{i}-2} / \mathbf{R} P^{2^{i}-2^{n+1}-2}\right)$ we see that $D^{*}$ is a $\mathbf{Z} / 2$-vector space on generators of the form $v^{I} t^{I^{\prime}} x^{j}$ with $l(I)=n+2$ and $2^{i-1}-2^{n} \leq j \leq 2^{i-1}-1$ modulo the relations $0=u_{n} x^{j}$ for $j \geq 2^{i-1}-2^{n}-n-2$. In particular, a homogeneous element of $D^{*}$ represented by an element of the form $\sum_{l(I)=n+2} \epsilon_{I, k} v^{I} t^{I^{\prime}} x^{2^{i-1}-k-1}$ with $\epsilon_{I, k} \in\{0,1\}$ and $\epsilon_{(n+2,0,0, \ldots), k}=0$ for all $k$ can be zero if and only if each $\epsilon_{I, k}$ is zero.

We are now ready for the induction argument, which will put severe restrictions on the canonical form for elements $u \in J_{2^{n+1}-2}\left(\mathbf{R} P^{2^{n+1}}\right)$ in Theorem 3.5.

5.2 Analysis of the leading terms .

We begin by observing that $u=2^{n+1} x_{2^{n+1}-1}$ satisfies the condition of the theorem. This is because $2^{n+1}\left(\eta_{R}\right)_{*}\left(x_{2^{n+1}-1}\right)$ and $2^{n+1}\left(\eta_{L}\right)_{*}\left(x_{2^{n+1}-1}\right)$ are S-dual to $2^{n+1} c(x)^{2^{i-1}-2^{n}}$ and $2^{n+1} x^{2^{i-1}-2^{n}}$, respectively. However,

$$
2^{n+1} c(x)^{2^{i-1}-2^{n}}=2^{n+1}\left(\sum_{v \geq 0} b_{v}^{B P} x^{v+1}\right)^{2^{i-1}-2^{n}},
$$

which is congruent to $2^{n+1} x^{2^{i-1}-2^{n}}$ modulo $<2^{n+2}, x^{2^{i-1}}>$, as required.

Therefore we may modify $u$ so that $\epsilon=0$ and we may write

$$
u=\sum_{l\left(I^{\prime}\right) \geq n+2, k} \epsilon_{I^{\prime}, k} v^{I^{\prime}} x_{2 k+1}
$$

with $2^{n+1}-\operatorname{deg}\left(v^{I^{\prime}}\right)-2=2 k$, whose $\mathrm{S}$-dual is

$$
D(u)=\sum_{l\left(I^{\prime}\right) \geq n+2, k} \epsilon_{I^{\prime}, k} v^{I^{\prime}} x^{2^{i-1}-k-1} .
$$

Note that this is also the expression for the S-dual of $\left(\eta_{L}\right)_{*}(u)$.

Next we shall compare the images in $E^{2^{i}-2^{n+1}}$ of the S-duals of $\left(\eta_{L}\right)_{*}(u)=$ $\sum_{l\left(I^{\prime}\right) \geq n+2, k} \epsilon_{I^{\prime}, k} v^{I^{\prime}} x_{2 k+1}$ and $\left(\eta_{R}\right)_{*}(u)$. To calculate the second image we need to sharpen Lemma 3.2. The proof of Lemma 3.2 easily yields, for $k \geq 1$,

$$
\left(\eta_{R}\right)_{*}\left(v_{k}\right)=2 t_{k}+\sum_{j \geq 1}^{k} v_{j} t_{k-j}^{2^{j}}\left(\operatorname{modulo} I^{2}\left[t_{1}, t_{2}, \ldots\right]\right) .
$$

Therefore, in $E^{2^{i}-2^{n+1}}$, the image of the S-dual of $\left(\eta_{R}\right)_{*}(u)$ is given by

$$
\sum_{I^{\prime}, k} \epsilon_{I^{\prime}, k} \prod_{j=1}^{r}\left(\sum_{a \geq 1} v_{a}\left(t_{j} x^{2^{a}-1}+t_{j-a}^{2^{a}}\right)\right)^{i_{j}} x^{2^{i-1}-k-1}\left(\sum_{q \geq 0} b_{q}^{B P} x^{q}\right)^{2^{i-1}-k-1},
$$

where $I^{\prime}=\left(i_{1}, \ldots, i_{r}\right)$ and $l\left(I^{\prime}\right)=n+2$. In this expression the multiple of $v_{1}^{n+2}$ is equal to

$$
\sum_{I^{\prime}, k} \epsilon_{I^{\prime}, k} v_{1}^{n+2} \prod_{j=1}^{r}\left(t_{j} x+t_{j-1}^{2}\right)^{i_{j}} x^{2^{i-1}-k-1}\left(\sum_{q \geq 0} b_{q}^{B P} x^{q}\right)^{2^{i-1}-k-1} .
$$


In $E^{*}$ the expression under consideration is unchanged by adding the following linear combination of multiples of $u_{n}$,

$$
\sum_{I^{\prime}, k} \epsilon_{I^{\prime}, k} u_{n} \prod_{j=1}^{r}\left(t_{j} x+t_{j-1}^{2}\right)^{i_{j}} x^{2^{i-1}-k-n-3}\left(\sum_{q \geq 0} b_{q}^{B P} x^{q}\right)^{2^{i-1}-k-1} .
$$

Hence, in $E^{2^{i}-2^{n+1}}, u$ is also represented by the expression

$$
\begin{aligned}
\sum_{I^{\prime}, k} & \epsilon_{I^{\prime}, k} \prod_{j=1}^{r}\left(\sum_{a \geq 1} v_{a}\left(t_{j} x^{2^{a}-1}+t_{j-a}^{2^{a}}\right)\right)^{i_{j}} x^{2^{i-1}-k-1}\left(\sum_{q \geq 0} b_{q}^{B P} x^{q}\right)^{2^{i-1}-k-1} \\
& -\sum_{I^{\prime}, k} \epsilon_{I^{\prime}, k} u_{n} \prod_{j=1}^{r}\left(t_{j} x+t_{j-1}^{2}\right)^{i_{j}} x^{2^{i-1}-k-n-3}\left(\sum_{q \geq 0} b_{q}^{B P} x^{q}\right)^{2^{i-1}-k-1} .
\end{aligned}
$$

Bearing in mind that $t_{0}=1=b_{0}^{B P}$, expanding this expression and collecting all the terms that do not involve any $t_{j}$ 's $(j \geq 1)$ gives an element representing the S-dual of $\left(\eta_{L}\right)_{*}(u)$ in $D^{2^{i}-2^{n+1}} \subseteq E^{2^{i}-2^{n+1}}$. Therefore, in $D^{2^{i}-2^{n+1}}$, expanding the expression and collecting all those monomials which do involve any of the $t_{j}$ 's should give zero. Also this expression contains no monomials of the form $v_{1}^{n+2} t_{1}^{s_{1}} \ldots t_{p}^{s_{p}} x^{q}$ so that we may apply the criterion of $\S 5.1$ to decide whether or not this element is zero in $D^{*}$.

Now consider the non-zero terms of smallest degree in $x$. That is, consider the maximal $k$ such that there exist $\epsilon_{I^{\prime}, k}$ 's which are non-zero. Since every term except $v_{1}^{n+2} x^{n+2}$ in $u_{n}$ has degree at least $n+3$, the terms of degree $2^{i-1}-k-1$ in $x$ in the modified representative for $u$ are given by

$$
\begin{gathered}
\sum_{I^{\prime}} \epsilon_{I^{\prime}, k} \prod_{j=1}^{r}\left(v_{j}+v_{j-1} t_{1}^{2^{j-1}}+\ldots+v_{1} t_{j-1}^{2}\right)^{i_{j}} x^{2^{i-1}-k-1} \\
-\sum_{I^{\prime}} \epsilon_{I^{\prime}, k} v_{1}^{n+2} \prod_{j=1}^{r}\left(t_{j-1}^{2}\right)^{i_{j}} x^{2^{i-1}-k-1} .
\end{gathered}
$$

Consider the subsum, for the same maximal $k$, over $I^{\prime}=\left(i_{1}, \ldots, i_{r}\right)$ such that $i_{2}, \ldots, i_{r-1}$ are not all zero and, of course, $i_{r}$ is non-zero. This means that $r \geq 3$. This subsum contributes

$$
\sum_{\left(i_{2}, \ldots, i_{r-1}\right) \neq \underline{0}} \epsilon_{I^{\prime}, k} v_{1}^{i_{1}+i_{r}} v_{2}^{i_{2}} \ldots v_{r-1}^{i_{r-1}} t_{r-1}^{2 i_{r}} x^{2^{i-1}-k-1} \in D^{*} .
$$

By induction on the maximal value of $r$ for which $\epsilon_{I^{\prime}, k}$ is non-zero in this subsum, we see that $\epsilon_{I^{\prime}, k}=0$ if $\left(i_{2}, \ldots, i_{r-1}\right) \neq \underline{0}$. This is because, for such terms, $v_{1}^{i_{1}+i_{r}} v_{2}^{i_{2}} \ldots v_{r-1}^{i_{r-1}} t_{r-1}^{2 i_{r}} x^{2^{i-1}-k-1}$ can only originate from $\epsilon_{I^{\prime}, k} v_{1}^{i_{1}} v_{2}^{i_{2}} \ldots v_{r-1}^{i_{r-1}} v_{i_{r}}^{i_{r}} x^{2^{i-1}-k-1}$.

So far then, we have shown that the terms of degree $2^{i-1}-k-1$ in $x$ in the modified represent ative for $\left(\eta_{R}\right)_{*}(u)$ are given by

$$
\sum_{I^{\prime}=\left(i_{1}, 0,0, \ldots, 0, i_{r}\right)} \epsilon_{I^{\prime}, k}\left(v_{1}^{i_{1}}\left(v_{r}+v_{r-1} t_{1}^{r^{-1}}+\ldots+v_{1} t_{r-1}^{2}\right)^{i_{r}}-v_{1}^{n+2} t_{r-1}^{2 i_{r}}\right) x^{2^{i-1}-k-1} \in D^{*}
$$

where $i_{1}+i_{r}=n+2$ in this sum. 
We write this representative for $\left(\eta_{R}\right)_{*}(u)$ as a sum of three parts in the form

$$
\begin{aligned}
& \quad \sum_{I^{\prime}=\left(i_{1}, 0,0, \ldots, 0, i_{r}\right), r \geq 4} \epsilon_{I^{\prime}, k}\left(v_{1}^{i_{1}}\left(v_{r}+v_{r-1} t_{1}^{2^{r-1}}+\ldots+v_{1} t_{r-1}^{2}\right)^{i_{r}}-v_{1}^{n+2} t_{r-1}^{2 i_{r}}\right) x^{2^{i-1}-k-1} \\
& +\sum_{I^{\prime}=\left(i_{1}, 0, i_{3}\right)} \epsilon_{I^{\prime}, k}\left(v_{1}^{i_{1}}\left(v_{3}+v_{2} t_{1}^{4}+v_{1} t_{2}^{2}\right)^{i_{3}}-v_{1}^{n+2} t_{r-1}^{2 i_{3}}\right) x^{2^{i-1}-k-1} \\
& +\sum_{I^{\prime}=\left(i_{1}, i_{2}\right)} \epsilon_{I^{\prime}, k}\left(v_{1}^{i_{1}}\left(v_{2}+v_{1} t_{1}^{2}\right)^{i_{2}}-v_{1}^{n+2} t_{1}^{2 i_{2}}\right) x^{2^{i-1}-k-1} .
\end{aligned}
$$

Next, in this simplified expression, consider the subsum

$$
\sum_{I^{\prime}=\left(i_{1}, 0,0, \ldots, 0, i_{r}\right), r \geq 4} \epsilon_{I^{\prime}, k}\left(v_{1}^{i_{1}}\left(v_{r}+v_{r-1} t_{1}^{2^{r-1}}+\ldots+v_{1} t_{r-1}^{2}\right)^{i_{r}}-v_{1}^{n+2} t_{r-1}^{2 i_{r}}\right) x^{2^{i-1}-k-1} \in D^{*} .
$$

Expanding out the first subsum and considering the terms

$$
\sum_{I^{\prime}=\left(i_{1}, 0,0, \ldots, 0, i_{r}\right), r \geq 4} \epsilon_{I^{\prime}, k} v_{1}^{i_{1}}\left(v_{r-1} t_{1}^{2^{r-1}}\right)^{i_{r}} x^{2^{i-1}-k-1} \in D^{*}
$$

shows that $\epsilon_{I^{\prime}, k}=0$ for each $I^{\prime}=\left(i_{1}, 0,0, \ldots, 0, i_{r}\right)$ with $r \geq 4$. This is because, in the simplified expression, $\epsilon_{\left(i_{1}, 0,0, \ldots, 0, i_{r}\right), k} v_{1}^{i_{1}}\left(v_{r-1} t_{1}^{2^{r-1}}\right)^{i_{r}} x^{2^{i-1}-k-1}$ can only originate from $\epsilon_{\left(i_{1}, 0,0, \ldots, 0, i_{r}\right), k} v_{1}^{i_{1}} v_{r}^{i_{r}} x^{2^{i-1}-k-1}$, if $r \geq 4$.

Thus we have shown that the terms of degree $2^{i-1}-k-1$ in $x$ in the modified representative for $\left(\eta_{R}\right)_{*}(u)$ are given by

$$
\begin{aligned}
& \sum_{i_{1}} \epsilon_{\left(i_{1}, 0, n+2-i_{1}\right), k}\left(v_{1}^{i_{1}}\left(v_{3}+v_{2} t_{1}^{4}+v_{1} t_{2}^{2}\right)^{\left(n+2-i_{1}\right)}-v_{1}^{n+2} t_{2}^{2\left(n+2-i_{1}\right)}\right) x^{2^{i-1}-k-1} \\
& +\sum_{i_{1}} \epsilon_{\left(i_{1}, n+2-i_{1}\right), k}\left(v_{1}^{i_{1}}\left(v_{2}+v_{1} t_{1}^{2}\right)^{n+2-i_{1}}-v_{1}^{n+2} t_{1}^{2 i_{2}}\right) x^{2^{i-1}-k-1} \\
& =\sum_{i_{1}} \epsilon_{\left(i_{1}, 0, n+2-i_{1}\right), k} \sum_{a=1}^{n+2-i_{1}}\left(\begin{array}{c}
n+2-i_{1} \\
a
\end{array}\right) v_{1}^{i_{1}}\left(v_{3}+v_{2} t_{1}^{4}\right)^{a}\left(v_{1} t_{2}^{2}\right)^{\left(n+2-i_{1}-a\right)} x^{2^{i-1}-k-1} \\
& +\sum_{i_{1}} \epsilon_{\left(i_{1}, n+2-i_{1}\right), k} \sum_{a=1}^{n+2-i_{1}}\left(\begin{array}{c}
n+2-i_{1} \\
a
\end{array}\right) v_{1}^{i_{1}} v_{2}^{a}\left(v_{1} t_{1}^{2}\right)^{n+2-i_{1}-a} x^{2^{i-1}-k-1} \\
& =\sum_{i_{1}} \epsilon_{\left(i_{1}, 0, n+2-i_{1}\right), k} \sum_{a=1}^{n+2-i_{1}}\left(\begin{array}{c}
n+2-i_{1} \\
a
\end{array}\right) v_{1}^{n+2-a}\left(v_{3}+v_{2} t_{1}^{4}\right)^{a} t_{2}^{2\left(n+2-i_{1}-a\right)} x^{2^{i-1}-k-1} \\
& +\sum_{i_{1}} \epsilon_{\left(i_{1}, n+2-i_{1}\right), k} \sum_{a=1}^{n+2-i_{1}}\left(\begin{array}{c}
n+2-i_{1} \\
a
\end{array}\right) v_{1}^{n+2-a} v_{2}^{a} t_{1}^{2\left(n+2-i_{1}-a\right)} x^{2^{i-1}-k-1} .
\end{aligned}
$$

By expanding this expression and considering the terms involving some $v_{3}$ 's and $t_{j}$ 's, we see that this expression can equal

$$
\sum_{i_{1}} \epsilon_{\left(i_{1}, 0, n+2-i_{1}\right)} v_{1}^{i_{1}} v_{3}^{n+2-i_{1}} x^{2^{i-1}-k-1}+\sum_{i_{1}} \epsilon_{\left(i_{1}, n+2-i_{1}\right)} v_{1}^{i_{1}} v_{2}^{n+2-i_{1}} x^{2^{i-1}-k-1} \in D^{*},
$$

the terms in the S-dual of $\left(\eta_{L}\right)_{*}(u)$ of degree $2^{i-1}-k-1$ in $x$, if and only if somewhere in the first sum $n+2-i_{1}=2^{\alpha}$ and the remaining $\epsilon_{\left(i_{1}, 0, n+2-i_{1}\right), k}$ must vanish. 
For the maximal $k$ under consideration there can be only one remaining such term, namely when $k=2^{n}-i_{1}-7 i_{3}-1=2^{n}-n-3-3 \cdot 2^{\alpha+1}$. Therefore we have whittled down the terms of degree $2^{i-1}-k-1$ in our representative for $\left(\eta_{R}\right)_{*}(u)$ to the form

$$
\begin{aligned}
& \epsilon_{\left(n+2-2^{\alpha}, 0,2^{\alpha}\right), k} v_{1}^{n+2-2^{\alpha}}\left(v_{3}^{2^{\alpha}}+v_{2}^{2^{\alpha}} t_{1}^{2^{\alpha+2}}\right) x^{2^{i-1}-k-1} \\
& +\sum_{i_{1}} \epsilon_{\left(i_{1}, n+2-i_{1}\right), k} \sum_{a=1}^{n+2-i_{1}}\left(\begin{array}{c}
n+2-i_{1} \\
a
\end{array}\right) v_{1}^{n+2-a} v_{2}^{a} t_{1}^{2\left(n+2-i_{1}-a\right)} x^{2^{i-1}-k-1} .
\end{aligned}
$$

To cancel the terms involving $t_{j}$ 's we must have either $\epsilon_{\left(n+2-2^{\alpha}, 0,2^{\alpha}\right), k}=0$ or there is a non-zero term with $a=2^{p h a}$ and $2\left(n+2-i_{1}-a\right)=2^{\alpha+2}$. However, this implies that $n+2-i_{1}=2^{\alpha+1}$ with the result that the binomial coefficient

$$
\left(\begin{array}{c}
n+2-i_{1} \\
a
\end{array}\right)=\left(\begin{array}{c}
2^{\alpha+1} \\
2^{\alpha}
\end{array}\right)
$$

is even.

Therefore the terms of degree $2^{i-1}-k-1$ in our representative for $\left(\eta_{R}\right)_{*}(u)$ take the form

$$
\sum_{i_{1}} \epsilon_{\left(i_{1}, n+2-i_{1}\right), k} \sum_{a=1}^{n+2-i_{1}}\left(\begin{array}{c}
n+2-i_{1} \\
a
\end{array}\right) v_{1}^{n+2-a} v_{2}^{a} t_{1}^{2\left(n+2-i_{1}-a\right)} x^{2^{i-1}-k-1}
$$

Similarly, by expanding this expression and considering the terms involving some $v_{2}$ 's and $t_{j}$ 's, we see that all the $\epsilon_{\left(i_{1}, n+2-i_{1}\right), k}$ must vanish except for possibly one for which $n+2-i_{1}=2^{\beta}$ and $k=2^{n}-i_{1}-3 i_{2}-1=2^{n}-n-3-2^{\beta+1}$. Therefore the terms of degree $2^{i-1}-k-1$ in our representative for $\left(\eta_{R}\right)_{*}(u)$ by now have the form

$$
\epsilon_{\left(n+2-2^{\beta}, 2^{\beta}\right), k} v_{1}^{n+2-2^{\beta}} v_{2}^{2^{\beta}} x^{2^{i-1}-k-1},
$$

which does equal the terms of degree $2^{i-1}-k-1$ in the S-dual of

$$
\left(\eta_{L}\right)_{*}\left(\epsilon_{\left(n+2-2^{\beta}, 2^{\beta}\right), k} v_{1}^{n+2-2^{\beta}} v_{2}^{2^{\beta}} x_{2 k+1}\right) .
$$

The following result recapitulates the progress of the induction argument so far.

Proposition 5.3. In Theorem 3.5, the element $u \in J_{2^{n+1}-2}\left(\mathbf{R} P^{\infty}\right)$ may be assumed to have the form

$$
\begin{aligned}
u & =\epsilon 2^{n+1} x_{2^{n+1}-1}+\epsilon_{\left(n+2-2^{\beta}, 2^{\beta}\right)} v_{1}^{n+2-2^{\beta}} v_{2}^{2^{\beta}} x_{2^{n+1}-2 n-2^{\beta+2}-5} \\
& +\sum_{\operatorname{deg}\left(v^{I^{\prime}}\right)>2 n+2^{\beta+2}+4} \epsilon_{I^{\prime}} v^{I^{\prime}} x_{2^{n+1}-\operatorname{deg}\left(v^{I^{\prime}}\right)-1} \\
& +\sum_{l\left(I^{\prime}\right) \geq n+3} \epsilon_{I^{\prime}} v^{I^{\prime}} x_{2^{n+1}-\operatorname{deg}\left(v^{I^{\prime}}\right)-1}
\end{aligned}
$$

with $\epsilon, \epsilon_{\left(n+2-2^{\beta}, 2^{\beta}\right)}, \epsilon_{I^{\prime}} \in\{0,1\}$. 


\section{Some important combinatorial identities.}

6.1. We are going to study $\left(\eta_{R}\right)_{*}\left(v_{1}^{i_{1}} \ldots v_{r}^{i_{r}} x^{2^{i-1}-k-1}\right)$ with $i_{1}+\ldots+i_{r}=n+2$, $2^{i-1}-k-1 \geq 2^{i-1}-2^{n}+n+2+2^{\beta+1}$ in the quotient of $E^{*}$ of $\S 5.1$ given by setting $0=t_{1}=t_{2}=\ldots=t_{j-1}=t_{j+1}=t_{j+2}=\ldots$. In fact, we shall therefore be in $D^{*} /<t_{1}, t_{2}, \ldots, t_{j-1}, t_{j+1}, t_{j+2}, \ldots>$. This is the vector space over $\mathbf{F}_{2}$ on a basis given by $v^{I} t_{j}^{\epsilon} x^{\delta}$ with $2^{i-1}-2^{n} \leq \delta \leq 2^{i-1}-1, l(I)=n+2$ and $I \neq(n+2,0,0, \ldots)$.

In order to compute these elements we shall need the following result.

Lemma 6.2. For $j \geq 1$, in

$$
\mathbf{Z}_{2}\left[v_{1}, v_{2}, \ldots, t_{1}, t_{2}, \ldots\right] /<2, v_{1}, v_{2}, \ldots, t_{1}, t_{2}, \ldots, t_{j-1}, t_{j+1}, \ldots>
$$

we have

$$
b_{v}^{B P} \equiv \begin{cases}t_{j}^{\left(2^{m j}-1\right) /\left(2^{j}-1\right)} & \text { if } v=2^{m j}-1 \\ 0 & \text { otherwise. }\end{cases}
$$

Proof. From $\$ 3.3$ we know that $b_{v}^{B P} \equiv 0$ unless $v=2^{s}-1$ and the congruence $0 \equiv \sum_{j=0}^{v} t_{j}\left(b_{2^{v-j}-1}^{B P}\right)^{2^{j}}$ (modulo $I \mathbf{Z}_{2}\left[v_{1}, \ldots, t_{1}, \ldots\right]$ ). Therefore, in the quotient of the statement of the lemma, $0 \equiv t_{j}\left(b^{B P} 2^{(m-1) j}-1\right)^{2^{j}}+t_{0} b_{2^{m j}-1}^{B P}$ so that, by induction, $b_{2^{m j}-1}^{B P} \equiv t_{j}^{1+2^{j}\left(2^{(m-1) j}-1\right) /\left(2^{j}-1\right)}=t_{k}^{\left(2^{m j}-1\right) /\left(2^{j}-1\right)}$, as required. It is easy to see that $b_{v}^{B P} \equiv 0$ in the other cases.

6.3. The case $j=1$.

Let $D^{*}$ be as in $\$ 5.1$. If $i_{1}+i_{2}<n+2$, Lemma 6.2 implies that in canonical form in $D^{*} /<t_{2}, t_{3}, t_{4}, \ldots>$

$$
\begin{gathered}
\left(\eta_{R}\right)_{*}\left(v_{1}^{i_{1}} \ldots v_{r}^{i_{r}} x^{j}\right)=\left(v_{1}+\sum_{k=1}^{\infty} v_{k} t_{1} x^{2^{k}-1}\right)^{i_{1}}\left(v_{2}+v_{1} t_{1}^{2}\right)^{i_{2}}\left(v_{3}+v_{2} t_{1}^{4}\right)^{i_{2}} \\
\ldots\left(v_{r}+v_{r-1} t_{1}^{2^{r-1}}\right)^{i_{r}} x^{j}\left(\sum_{v=0}^{\infty} t_{1}^{2^{v}-1} x^{2^{v}-1}\right)^{j} .
\end{gathered}
$$

If $i_{1}+i_{2}=n+2$ we have

$$
\begin{aligned}
\left(\eta_{R}\right)_{*}\left(v_{1}^{n+2-i_{2}} v_{2}^{i_{2}} x^{j}\right)= & \left(\left(v_{1}+\sum_{k=1}^{\infty} v_{k} t_{1} x^{2^{k}-1}\right)^{n+2-i_{2}}\left(v_{2}+v_{1} t_{1}^{2}\right)^{i_{2}}\right. \\
& \left.-\left(\sum_{k=1}^{\infty} v_{k} x^{2^{k}-2}\right)^{n+2}\left(1+x t_{1}\right)^{n+2-i_{2}} t_{1}^{2 i_{2}}\right) x^{j}\left(\sum_{v=0}^{\infty} t_{1}^{2^{v}-1} x^{2^{v}-1}\right)^{j} .
\end{aligned}
$$

Now suppose that, in (cohomological) dimension $2^{i}-2^{n+1}$, we have an element as in Proposition 5.3 but with $\epsilon=0$ in the leading term. Such an element has an S-dual of the form

$$
D(u)=\sum_{I} \epsilon_{I} v^{I} x^{2^{i-1}-k-1}
$$


with $\epsilon_{I} \in\{0,1\}$ and where the lowest degree terms in $x$ consist solely of the term $v_{1}^{n+2-2^{\beta}} v_{2}^{2^{\beta}} x^{2^{i-1}-2^{n}+n+2+2^{\beta+1}}$. This element satisfies $\left(\eta_{R}\right)_{*}(D(u))=D(u)$ in $D^{*} /<t_{2}, t_{3}, t_{4}, \ldots>$. We may obtain relations in $\mathbf{Z} / 2\left[x, t_{1}\right] /\left(x^{2^{n}-2-2 j}\right)$ by equating coefficients of $v_{1}^{n+2-j} v_{2}^{j}$. In fact, these relations lie in the subring, $\mathbf{Z} / 2[w] /\left(w^{2^{n}-2-2 j}\right)$, where $w=x t_{1}$. Since $j \leq n+2$ we may view all these equations as being in the same ring. For example $\mathbf{Z} / 2[w] /\left(w^{2^{n}-3 n-6}\right)$ will suit our purposes providing that $n \geq 5$.

Now consider all those terms whose $\left(\eta_{R}\right)_{*}$-image contains some monomials of the form $v_{1}^{i_{1}} v_{2}^{i_{2}} x^{s} t_{1}^{r}$. These can only come from monomials with $I=\left(i_{1}, i_{2}, i_{3}\right)$. A calculation of the monomials of the form $v_{1}^{n+2-2^{\beta}} v_{2}^{2^{\beta}} x^{a} t_{1}^{b}$ occurring in

$$
\left(\eta_{R}\right)_{*}\left(v_{1}^{i_{1}} v_{2}^{i_{2}} v_{3}^{i_{3}} x^{2^{i-1}-2^{n}+n+2+2 i_{2}+6 i_{3}}\right),
$$

where $n+2=i_{1}+i_{2}+i_{3}$, together with some elementary combinatorics, yields the following result.

Proposition 6.4. Set $w=x t_{1}$ and $L(w)=\sum_{v=0}^{\infty} t_{1}^{2^{v}-1} x^{2^{v}-1}=\sum_{v=0}^{\infty} w^{2^{v}-1}$. In the notation of Proposition 5.3, if $j \leq n+2$, then

$$
\begin{aligned}
& \epsilon_{(n+2-j, j)} \\
& =\sum_{\left(i_{1}, i_{2}, i_{3}\right), i_{1}+i_{2}+i_{3}=n+2, b} L(w)^{2^{i-1}-2^{n}+n+2+2 i_{2}+6 i_{3}}\left(\begin{array}{c}
n+2-i_{2}-i_{3} \\
b
\end{array}\right)\left(\begin{array}{c}
i_{2} \\
i_{2}+i_{3}+b-j
\end{array}\right) \\
& \quad \times \epsilon_{\left(i_{1}, i_{2}, i_{3}\right)}(1+w)^{n+2-i_{2}-i_{3}}\left(\frac{w}{1+w}\right)^{b}\left(w^{2}\right)^{i_{2}+i_{3}+b-j} w^{4 i_{3}} \\
& \quad-\sum_{\left(i_{1}, i_{2}\right), i_{1}+i_{2}=n+2,} L(w)^{2^{i-1}-2^{n}+n+2+2 i_{2}} \epsilon_{\left(i_{1}, i_{2}\right)}\left(\begin{array}{c}
n+2 \\
j
\end{array}\right)(1+w)^{n+2-i_{2}} w^{2 i_{2}}
\end{aligned}
$$

in $\mathbf{Z} / 2[w] /\left(w^{2^{n}-3 n-6}\right)$.

6.5. The case $j=2$.

Next we calculate the monomials of the form $v_{1}^{n+2-j} v_{2}^{j} x^{a} t_{2}^{b}$ occurring in

$$
\left(\eta_{R}\right)_{*}\left(v_{1}^{i_{1}} v_{2}^{i_{2}} \ldots v_{r}^{i_{r}} x^{s}\right) \in D^{2^{i}-2^{n+1}} /<t_{1}, t_{3}, t_{4}, \ldots>
$$

Since

$$
\left(\eta_{R}\right)_{*}\left(v_{s}^{i_{s}}\right) \equiv\left(\sum_{a \geq 1} v_{a}\left(t_{s} x^{2^{a}-1}+t_{s-a}^{2^{a}}\right)\right)^{i_{s}}
$$

the term under consideration can only come from monomials with $I=\left(i_{1}, i_{2}, i_{3}, i_{4}\right)$.

In this case one obtains the following identity.

Proposition 6.6. Set $w^{3}=t_{2} x^{3} \quad$ and $\quad M(w)=\sum_{m \geq 0} t_{2}^{\left(2^{2 m}-1\right) /\left(2^{2}-1\right)} x^{2^{2 m}-1}=$ $\sum_{m \geq 0} w^{\left(2^{2 m}-1\right)}$. In the notation of Proposition 5.3, if $j \leq n+2$, then 


$$
\begin{aligned}
\epsilon_{n+2-j, j}= & \sum_{\left(i_{1}, i_{2}, i_{3}, i_{4}\right), i_{1}+i_{2}+i_{3}+i_{4}=n+2} \epsilon_{\left(i_{1}, i_{2}, i_{3}, i_{4}\right)}\left(\begin{array}{c}
i_{2} \\
j-i_{4}
\end{array}\right) \\
& \times\left(1+w^{3}\right)^{j-i_{4}} w^{3\left(i_{2}+2 i_{3}+5 i_{4}-j\right)} M(w)^{2^{i-1}-2^{n}+n+2+2 i_{2}+6 i_{3}+14 i_{4}} \\
& -\sum_{\left(i_{1}, i_{2}, i_{3}\right), i_{1}+i_{2}+i_{3}=n+2} \epsilon_{\left(i_{1}, i_{2}, i_{3}\right)}\left(\begin{array}{c}
n+2 \\
j
\end{array}\right) w^{3\left(i_{2}+2 i_{3}\right)} M(w)^{2^{i-1}-2^{n}+n+2+2 i_{2}+6 i_{3}}
\end{aligned}
$$

in $\mathbf{Z} / 2[w] /\left(w^{\left(2^{n}-3 n-6\right)}\right)$.

6.7. The case $j \geq 3$.

If, rather than $t_{1}$ or $t_{2}$, we try this process with $t_{k}$ for $k \geq 3$ we obtain the identity below.

Proposition 6.8. Set $w^{2^{k}-1}=t_{k} x^{2^{k}-1}$ and $M_{k}(w)=\sum_{m \geq 0} t_{k}^{\left(2^{m k}-1\right) /\left(2^{k}-1\right)} x^{2^{m k}-1}=$ $\sum_{m \geq 0} w^{\left(2^{m k}-1\right)}$. In the notation of Proposition 5.3, if $j \leq n+2$ and $k \geq 3$, then

$$
\begin{aligned}
& \epsilon_{(n+2-j, j)} \\
& =\left(\sum_{\left(i_{1}, i_{2}, 0, \ldots, i_{k}, i_{k+1}, i_{k+2}\right), i_{1}+i_{2}+i_{k}+i_{k+1}+i_{k+2}=n+2} \epsilon_{\left(i_{1}, \ldots\right)}\left(\begin{array}{c}
i_{k} \\
j-i_{2}-i_{k+2}
\end{array}\right) w^{\left(2^{k}-1\right)\left(i_{k}+2 i_{k+1}+4 i_{k+2}\right)}\right. \\
& \left.\quad-\sum_{\left(i_{1}, 0, \ldots, i_{k}, i_{k+1}\right), i_{1}+i_{k}+i_{k+1}=n+2} \epsilon_{\left(i_{1}, \ldots\right)}\left(\begin{array}{c}
n+2 \\
j
\end{array}\right) w^{\left(2^{k}-1\right)\left(i_{k}+2 i_{k+1}\right)}\right) \\
& \quad \times M_{k}(w)^{2^{i-1}-2^{n}+n+2+2 i_{2}+\left(2^{k}-2\right) i_{k}+\left(2^{k+1}-2\right) i_{k+1}+\left(2^{k+2}-2\right) i_{k+2}}
\end{aligned}
$$

in $\mathbf{Z} / 2[w] /\left(w^{2^{n}-3 n-6}\right)$.

REMARK 6.9. In using the identities of Propositions 6.4, 6.6 and 6.8 it is sometimes convenient to add them over all values of $j$.

\section{Theorem 3.5-the final step.}

7.1. To complete the proof of Theorem 3.5 we must show that $\epsilon_{\left(n+2-2^{\beta}, 2^{\beta}\right)}=0$ in Proposition 5.3. Since the $\epsilon_{I}$ 's are equal to 0 or 1 it suffices to show that the identities of Propositions 6.4, 6.6 and 6.8 imply that $\epsilon_{\left(n+2-2^{\beta}, 2^{\beta}\right)} \equiv 0$ (modulo 2). Since the algebra is very laborious we shall merely sketch the procedure.

In the circumstances of Theorem 3.5 we may suppose, by Proposition 5.3, that $\epsilon=0$ (subtracting $2^{n+1} x_{2^{n+1}-1}$ from $u$ if necessary, as in $\$ 5.2$ ) and that $\epsilon_{\left(n+2-2^{j}, 2^{j}\right)}=0$ for $0 \leq j \leq \beta-1$. Then the first step of the induction shows that the lowest $x$ degree in the S-dual, $D(u)$, is $2^{i-1}-2^{n}+n+2+2^{\beta+1}$, which means that $\epsilon_{\left(i_{1}, i_{2}, i_{3}\right)}=0$ for all $i_{1}+i_{2}+i_{3}=n+2$ and $2 i_{2}+6 i_{3} \leq 2^{\beta+1}$ - except possibly $\epsilon_{\left(n+2-2^{\beta}, 2^{\beta}\right)}$, which we are trying to show is zero in order to complete the last step of the main induction.

The Arf invariant of framed manifolds is well understood when $n=1,2,3,4$ so that we shall assume that $n \geq 5$, which ensures that $8 \leq 2^{n}-2 n-6$. From Proposition 6.4 modulo $w^{8} \mathbf{Z} / 2[w] /\left(w^{2^{n}-3 n-6}\right)$, setting $j=1,2,3,4,5,6$ successively yields 
$\epsilon_{(n-1,1)}=0=\epsilon_{(n-2,2)}=\epsilon_{(n-3,3)}$ and then Proposition 6.6 modulo $w^{8} \mathbf{Z} / 2[w] /\left(w^{2^{n}-3 n-6}\right)$ yields $\epsilon_{(n-4,4)}=0$.

Therefore we may assume that $n \geq 5$ and $\beta \geq 3$.

We shall sketch the remainder of the proof for the case in which $n$ is odd. In this case its suffices to study the identities of Propositions 6.4, 6.6 and 6.8 modulo $w^{8} \mathbf{Z} / 2[w] /\left(w^{2^{n}-3 n-6}\right)$. The case when $n$ is even is similar but more complicated.

When $n$ is odd Proposition 6.6 implies that $\epsilon_{(n+2-2 s, 2 s)}=\epsilon_{(n+1-2 s, 2 s+1)}$ for all $s$. Setting $j=2^{\beta}-1,2^{\beta}, 2^{\beta}+1$ in Propositions 6.4 and 6.6 yields $\epsilon_{\left(n-2^{\beta}+3,2^{\beta}-2,1\right)}=$ $0=\epsilon_{\left(n-2^{\beta}+2,2^{\beta}-1,1\right)}=\epsilon_{\left(n-2^{\beta}, 2^{\beta}+1,1\right)}, \quad \epsilon_{\left(n+1-2^{\beta}, 2^{\beta}, 1\right)}=\epsilon_{\left(n+2-2^{\beta}, 2^{\beta}\right)} \quad$ and $\quad \epsilon_{\left(n-2^{\beta}, 2^{\beta}+2\right)}=$ $\epsilon_{\left(n-2^{\beta}+2,2^{\beta}\right)}$ if $n \equiv 1$ (modulo 4) while $\epsilon_{\left(n-2^{\beta}, 2^{\beta}+2\right)}=0$ if $n \equiv 3$ (modulo 4). In addition, Proposition 6.8 modulo $w^{8} \mathbf{Z} / 2[w] /\left(w^{2^{n}-3 n-6}\right)$ yields the congruence $0 \equiv \epsilon_{(n+1-j, j, 1)}+$ $\epsilon_{(n+2-j, j-1,1)}+\epsilon_{(n+2-j, j)}$ (modulo 2) for odd $n$.

When $n \equiv 1$ (modulo 4) these identities together with Proposition 6.6 for $j=2^{\beta}+1$ yield $\epsilon_{\left(n+2-2^{\beta}, 2^{\beta}\right)}=0$, completing the proof in that case. When $n \equiv 3$ (modulo 4) setting $j=2^{\beta}+3,2^{\beta}+4,2^{\beta}+5$ yields $\epsilon_{\left(n-2^{\beta}-4,2^{\beta}+6\right)}=0=\epsilon_{\left(n-2^{\beta}-2,2^{\beta}+4\right)}$ and

$$
\epsilon_{\left(n-2^{\beta}-3,2^{\beta}+4,1\right)}=\epsilon_{\left(n-2^{\beta}-2,2^{\beta}+4\right)} \equiv\left(\begin{array}{c}
n+2 \\
4
\end{array}\right) \epsilon_{\left(n-2^{\beta}+2,2^{\beta}\right)} \text { (modulo 2) }
$$

from which one sees that $\epsilon_{\left(n+2-2^{\beta}, 2^{\beta}\right)}=0$ if $n \equiv 3$ (modulo 8 ).

Finally, if $n \equiv 7$ (modulo 8 ), one shows by setting $j=2^{\beta}+7,2^{\beta}+8, \ldots$ that $\epsilon_{\left(n+2-2^{\beta}, 2^{\beta}\right)}=\epsilon_{\left(n+1-2^{\beta}, 2^{\beta}, 1\right)}$ are the only possibly non-zero coefficients of length two or three. Summing over $j$ the identity of Proposition 6.8 with $k=3$, as in Remark 6.9, we obtain in $\mathbf{Z} / 2[w] /\left(w^{2^{n}-3 n-6}\right)$

$$
\begin{aligned}
\epsilon_{\left(n+2-2^{\beta}, 2^{\beta}\right)}= & \sum_{j} \epsilon_{(n+2-j, j)} \\
= & \sum_{\left(i_{1}, i_{2}, 0, i_{4}, i_{5}\right), i_{1}+i_{2}+i_{4}+i_{5}=n+2 \epsilon_{\left(i_{1}, i_{2}, 0, i_{4}, i_{5}\right)}}\left(w^{7}\right)^{2 i_{4}+4 i_{5}} \\
& \times\left(1+w^{7}+w^{2^{6}-1}+w^{2^{9}-1}+\ldots\right)^{2^{i-1}-2^{n}+n+2+2 i_{2}+\left(2^{4}-2\right) i_{4}+\left(2^{5}-2\right) i_{5}} \\
= & \epsilon_{\left(n+2-2^{\beta}, 2^{\beta}\right)}\left(1++w^{7}+O\left(w^{2^{6}-1}\right)\right)+O\left(\left(w^{7}\right)^{2}\right) .
\end{aligned}
$$

Considering the coefficient of $w^{7}$ shows that $\epsilon_{\left(n+2-2^{\beta}, 2^{\beta}\right)}=0$ when $n \equiv 7$ (modulo 8).

\section{REFERENCES}

1. J. F. Adams, Stable homotopy and generalised homology (University of Chicago Press, 1974).

2. J. F. Adams, Lectures on generalized cohomology, Lecture Notes in Mathematics No. 99 (Springer-Verlag, 1969).

3. M. G. Barratt, J. D. S. Jones and M. Mahowald, The Kervaire invariant and the Hopf invariant in Proc. Conf. Algebraic Topology, Seattle, Lecture Notes in Mathematics No. 1286 (Springer-Verlag, 1987).

4. W. Browder, The Kervaire invariant of framed manifolds and its generalizations, Ann. of Math. 90 (1969), 157-186.

5. D. Husemoller, Fibre bundles 2nd edition (Springer-Verlag, 1975). 
6. D. S. Kahn and S. B. Priddy, The transfer and stable homotopy theory, Proc. Camb. Phil. Soc. 83 (1978), 103-112.

7. M. Kervaire, A manifold which does not admit any differentiable structure, Comment. Math. Helv. 34 (1960), 257-270.

8. J. Klippenstein and V.P. Snaith, A conjecture of Barratt-Jones-Mahowald concerning framed manifolds having Kervaire invariant one, Topology (4) 27 (1988) 387-392.

9. K. Knapp, Im(J)-theory and the Kervaire invariant, Math. Z. 226 (1997), 103-125.

10. H. R. Miller, D. C. Ravenel and W.S . Wilson, Periodic phenomena in the AdamsNovikov spectral sequence, Ann. of Math. 106 (1977), 469-516.

11. S. P. Novikov, The methods of algebraic topology from the viewpoint of cobordism theories (Russian) Izvestija Akademii Nauk. S.S.S.R. Serija Mat. 31 (1967), 855-951.

12. V. P. Snaith and J. Tornehave, On $\pi_{*}(B O)$ and the Arf invariant of framed manifolds, Contemp. Math. 12 (1989), 299-313. (1979).

13. V. P. Snaith, Algebraic cobordism and K-theory, Mem. Amer. Math. Soc. No. 221

14. C. Soulé, Éléments cyclotomiques en K-théorie, Soc. Math. de France Astérisque 1478 (1987), 225-257.

15. N. E. Steenrod, Cohomology operations, Annals Math. Studies No. 50 (written and revised by D. B. A. Epstein) (Princeton University Press, 1962).

16. W. S. Wilson, A BP-introduction and sampler, CBMS Regional Conf. Series in Math., No. 48 (Amer. Math. Soc., Providence, R.I., 1982). 